J. D. Donald and F. J. Flanigan

Nagoya Math. J.

Vol. 57 (1975), 107-119

\title{
DEFORMATION METHODS AND THE STRONG UNBOUNDED REPRESENTATION TYPE OF $p$-GROUPS
}

\author{
J. D. DONALD AND F. J. FLANIGAN
}

\section{Introduction.}

A basic problem in the representation theory of a finite group $G$ is the determination of all indecomposable $G$-modules. Thus, for $G=C(n)$ = a cyclic group of order $n$ over an arbitrary field, the indecomposable representations, finite in number, are known from the theory of a single linear transformation. In 1954 Higman [9] showed that, in sharp contrast to the classical case of characteristic zero, an arbitrary finite group $G$ has indecomposables of arbitrarily high dimension over any field of prime characteristic $p$ iff the $p$-Sylow subgroup of $G$ is non-cyclic (cf. unbounded representation type [3, p. 431]). Examples published by Heller and Reiner [8] in 1961 indicated that this phenomenon is even more extensive; reinterpreting a result of Dieudonné [4] as classifying the indecomposable modules for a square zero algebra on two generators, they showed that $G=C(p) \times C(p)$ (and therefore many other groups) has infinitely many non-isomorphic indecomposables in every even dimension over an infinite field of characteristic $p$ (cf. strong unbounded representation type). At present, all $C(p) \times C(p)$ indecomposables are known only in the case $p=2$, the result also being given (essentially) in [4] (cf. also [1], [2], [12]). In particular, the four-group $C(2) \times C(2)$ affords only two (dual) indecomposable representations in each odd dimension $\geq 3$.

This paper contributes, by way of examples and a suggested technique, to a fuller description of this plethora of $G$-modules. Our study of the deformation of algebra representations [5], [6], [7], when brought to bear on the Heller-Reiner modules for a non-cyclic abelian $p$-group $G$, has led us to these observations:

Received May 13, 1974.

Revised October 25, 1974. 
(1) If $G$ is not the four-group, then it has infinitely many indecomposables in each odd dimension $\geq 3$ also, provided of course that the field $k$ is infinite (Section 2).

(2) For $p=2$, the odd-dimensional Heller-Reiner indecomposables for the four-group $G$ must decompose in every nontrivial generic deformation and are actually rigid as modules for the square zero algebra on two generators. This phenomenon may be viewed as the deformationtheoretic counterpart of the fact (see above) that there are no other indecomposables but these two. We partially re-obtain this finiteness result in the course of straightening-out some deformations (Section 4).

(3) A result recently announced by Janusz ([10], [11]) that almost all non-cyclic abelian $p$-groups have infinitely many faithful indecomposables in every sufficiently large dimension is obtained by a slight extension of our methods (Section 3).

Actually our results grew out of a deceptive intuition. We speculated that if an indecomposable module were not rigid (as are the principal indecomposables and the irreducibles), then a generic deformation of that module would provide a parametrized family of non-isomorphic indecomposables of the same dimension. Thus we began by rediscovering the Heller-Reiner modules referred to above and trying to deform them. The even-dimensional family is in fact obtained as a deformation of a particularly simple member of it. Secondly, we found a common deformation of the two odd-dimensional types (no longer factoring through an algebra with square zero radical) valid for $G=C\left(p^{e}\right) \times C\left(p^{f}\right)$ except $C(2) \times C(2)$. It is easy to prove the indecomposability of all these modules directly (see (1) above). Subsequently we extended our methods slightly to include Janusz' faithfulness result ((3) above).

These examples lent support to our potentially very useful deformation principle, but then we discovered a counterexample (cf. (5.2)) a generic deformation of an indecomposable need not be indecomposable. Nonetheless, we suspect that every indecomposable over an algebraically closed field arises by deforming one defined over the prime field. As an illustration of the usefulness of a deformation-theoretic approach, we mention that the rigidity and straightening-out theorems (cf. [6]) imply that certain modules cannot belong to a larger parametrized family!

Section 1 briefly describes the Heller-Reiner modules. Then we begin to deform. Section 2 gives the common deformation of the two odd- 
dimensional classes. Section 3 describes a simple way of extending these results to yield faithful representations of groups with exponent $>p$. Section 4 contains a rigidity result for $C(2) \times C(2)$-modules and Section 5 contains some examples of deformations. It is only in these last two sections that any technical deformation-theoretic language appears.

\section{The Heller-Reiner modules.}

(1.1) We list here, using a convenient notation, various indecomposable representations for a non-cyclic multiplicative abelian group $G=\prod_{i=1}^{i=m} C\left(p^{e_{i}}\right)$ with basis $a_{1}, \cdots, a_{m}, m \geq 2, e_{i} \geq 1$, over a scalar field $k$ of prime characteristic $p$. See [8].

The group algebra $k G$ has radical of codimension one. We will define the action of the $m$ generators $a_{i}-1$ of the radical of $k G$ so that all products $\left(a_{i}-1\right)\left(a_{j}-1\right)$ will act as 0 . This is sufficient to define a "height two" representation of $k G$ on a $k$-space $V$, that is,

$$
V>(\operatorname{rad} k G) V>(\operatorname{rad} k G)^{2} V=(0) .
$$

In each case let the $k$-space $V=X \oplus Y$ with bases $\left\{x_{1}, \cdots, x_{\beta}\right\}$, $\left\{x_{\beta+1}, \cdots, x_{N}\right\}$ for $X, Y$. We always put $\left(a_{i}-1\right) Y=(0)$ for all $i$, and $\left.\left(a_{i}-1\right)\right|_{X} \in \operatorname{Hom}_{k}(X, Y)$. With one exception, (1.2), we do not specify the $a_{i}-1$ for $i \geq 3$.

(1.2) The first odd-dimensional class: Let $\beta=n \geq 1, N=2 n+1$ and, for $1 \leq r \leq n$

$$
\left(a_{1}-1\right)\left(x_{r}\right)=x_{r+n}, \quad\left(a_{2}-1\right)\left(x_{r}\right)=x_{r+n+1}
$$

Note that if $m \geq 3$, then the choices

$$
\left(a_{3}-1\right)\left(x_{1}\right)=t x_{n+1}, t \in k, \quad\left(a_{3}-1\right)\left(x_{r}\right)=0, \quad 2 \leq r \leq n,
$$

yield inequivalent representations, since for $\alpha \in k, \operatorname{dim}_{k} \operatorname{ker}\left[\alpha\left(a_{1}-1\right)-\right.$ $\left.\left(a_{3}-1\right)\right]=n+2$ iff $\alpha=t$. This provides "many" indecomposables in the special case $G$ is an elementary abelian $p$-group $C(p) \times C(p) \times \cdots \times$ $C(p)$ of dimension $m>2$. (Contrast the case $p=2, m=2$.) We use this in (3.2).

(1.3) The second odd-dimensional class: These are the duals to (1.2). Let $\beta=n+1 \geq 2, N=2 n+1$, and

$$
\left(a_{1}-1\right)\left(x_{1}\right)=0, \quad\left(a_{1}-1\right)\left(x_{r}\right)=x_{r+n}, \quad 2 \leq r \leq n+1,
$$




$$
\left(a_{2}-1\right)\left(x_{r}\right)=x_{r+n+1}, \quad 1 \leq r \leq n, \quad\left(a_{2}-1\right)\left(x_{n+1}\right)=0 .
$$

Remark. In [8] it is pointed out that, for $G=C\left(p^{e}\right) \times C\left(p^{f}\right)$ (thus $m=2$ ), every height two odd-dimensional $k G$-indecomposable is either (1.2) or (1.3).

(1.4) An even-dimensional class: Let $\beta=n \geq 1, N=2 n$, and

$$
\begin{aligned}
& \left(a_{1}-1\right)\left(x_{r}\right)=x_{r+n}, \quad 1 \leq r \leq n \\
& \left(a_{2}-1\right)\left(x_{r}\right)=x_{r+n+1}+t x_{r+n}, \quad 1 \leq r \leq n-1 \\
& \left(a_{2}-1\right)\left(x_{n}\right)=t x_{2 n}, \quad t \in k
\end{aligned}
$$

These representations are inequivalent for distinct $t \in k$ since for $\alpha \in k$, $\operatorname{dim}_{k} \operatorname{ker}\left[\alpha\left(\alpha_{1}-1\right)-\left(a_{2}-1\right)\right]=n+1$ iff $\alpha=t$. Consequently an infinite field $k$ affords infinitely many distinct even-dimensional indecomposables for $G$.

In [8] it is pointed out that, for groups $G=C\left(p^{e}\right) \times C\left(p^{f}\right)$ (thus $m=2$ ), every even-dimensional height two $k G$-indecomposable is of the type (1.4) iff $k$ is algebraically closed.

(1.5) Faithfulness of these representations when all $e_{i}=1$. Note that each group element $a_{i}^{r}$ acts as $r\left(a_{i}-1\right)+1$. Thus, if $G=C(p) \times$ $C(p)$ is elementary abelian with $m=2$, then the above representations are faithful for $G$.

In general, since

$$
\operatorname{dim}_{Z / p Z} \operatorname{Hom}_{k}(X, Y)=\operatorname{dim}_{k} X \cdot \operatorname{dim}_{k} Y \cdot[k: Z / p Z],
$$

proper choices of the radical generators $a_{i}-1 \in \operatorname{Hom}_{k}(X, Y)$ with $3 \leq i$ $\leq m$ will yield faithful representations of an elementary abelian $G$ provided the field $k$ is large enough.

See Section 3 for the case where some $e_{i}>1$.

\section{The common deformation of the two odd-dimensional classes.}

(2.1) Now we show that an infinitude of odd-dimensional indecomposable $G$-modules, which occured at height two in the even-dimensional case (1.4), occurs at height three. Our method is to write down a oneparameter family $V_{t}$ of deformations of (1.2) and observe that the generic member is indecomposable. The modules (1.2) and (1.3) are the cases $t=0$ and $t=1$ of this family.

We require $G=\prod_{i=1}^{i=m} C\left(p^{e_{i}}\right)$ with $p^{e_{1}} \neq 2, m \geq 2$, and char $k=p$. 
(2.2) The G-modules $V_{t}$. Let $V=X \oplus Y$ with $k$-bases $\left\{x_{1}, \cdots, x_{n}\right\}$ and $\left\{x_{n+1}, \cdots, x_{2 n+1}\right\}$ for $X$ and $Y$ as in (1.2). We have $a_{1}^{2} \neq 1$ in $G$. Fix $t \in k$ and define the action of $a_{1}-1$ by

$$
\begin{array}{ll}
\left(a_{1}-1\right)\left(x_{1}\right)=(1-t) x_{n+1}, & \left(a_{1}-1\right)\left(x_{r}\right)=x_{r+n}, \quad 2 \leq r \leq n \\
\left(a_{1}-1\right)\left(x_{n+1}\right)=t x_{2 n+1}, & \left(a_{1}-1\right)\left(x_{r}\right)=0, \quad n+2 \leq r \leq 2 n+1 .
\end{array}
$$

The action of $a_{2}-1$ is defined by

$$
\left(a_{2}-1\right)\left(x_{r}\right)=x_{r+n+1}, \quad 1 \leq r \leq n, \quad\left(a_{2}-1\right)(Y)=(0) .
$$

Moreover, for $3 \leq i \leq m$ we require that $\left.\left(a_{i}-1\right)\right|_{X} \in \operatorname{Hom}_{k}(X, Y)$ and also that $\left(a_{i}-1\right)(Y)=(0)$.

Now one notes that, except for $\left(a_{1}-1\right)^{2}\left(x_{1}\right)=t(1-t) x_{2 n+1}$, defining all products $\left(a_{i}-1\right)\left(a_{j}-1\right)$ and so on to act as zero yields a representation of $k G$ on the space $X \oplus Y$. We denote this module $V_{t}$. It is immediate that $t=0$ and $t=1$ reduce to (1.2) and (1.3) respectively.

(2.3) $V_{t}$ is indecomposable. We sketch the argument. It is standard, and applies in Section 1 also; see [3, p. 433].

Note first that a nonzero $k G$-direct summand cannot be contained in $Y$. Now, if $X_{0}$ is any $k$-space complement to $Y$ in $V_{t}$, and if we have a nontrivial $k$-space decomposition $X_{0}=X_{1} \oplus X_{2}$, then one checks that $\operatorname{dim}_{k}(\operatorname{rad} k G) X_{j}>\operatorname{dim}_{k} X_{j}$ for $j=1,2$. (This follows from the fact that $a_{1}-1, a_{2}-1 \operatorname{map} X_{0} \rightarrow Y$ injectively.) Thus, $\operatorname{dim}_{k}\left(X_{1} \oplus X_{2}\right)=n$ and the hypothesis $(\operatorname{rad} k G) X_{1} \cap(\operatorname{rad} k G) X_{2}=(0)$ would imply $n+1=\operatorname{dim}_{k} Y \geq$ $n+2$, a contradiction. Thus $X_{1}$ and $X_{2}$ cannot generate complementary submodules of $V_{t}$. The assertion follows.

(2.4) Let $t \neq 0,1$ in $k$. Then $V_{t}$ and $V_{s}$ are kG-isomorphic iff $s=t$ or $1-t$. To prove this, suppose first $s=1-t$ and $t \neq 0,1$. Define a $k$-linear map $\phi: V_{t} \rightarrow V_{1-t}$ on the basis $x_{1}, \cdots, x_{n+1}, \cdots, x_{2 n+1}$ by

$$
\phi\left(x_{i}\right)=x_{i}, i \neq n+1, \quad \phi\left(x_{n+1}\right)=t(1-t)^{-1} x_{n+1}
$$

One readily checks that $\phi$ yields the desired $k G$-isomorphism.

Conversely, in $V_{t}$ we have $(\operatorname{rad} k G)^{2} V_{t}=k$-span $\left\{x_{2 n+1}\right\}$. Moreover, since $a_{2}-1$ gives a $k$-space isomorphism $X \rightarrow k$-span $\left\{x_{n_{+2}}, \cdots, x_{2 n_{+1}}\right\}$, we may consider the inverse map here (denoted $\left.\left(a_{2}-1\right)^{-1}\right)$ and obtain the equation

$$
\left(a_{1}-1\right)\left[\left(a_{1}-1\right)\left(a_{2}-1\right)^{-1}\right]^{n}\left(x_{2 n+1}\right)=t(1-t) x_{2 n+1}
$$


Thus a certain intrinsically defined map on the canonical subspace $(\operatorname{rad} k G)^{2} V_{t}$ is multiplication by $t(1-t)$. It follows that $V_{t}$ isomorphic to $V_{s}$ forces $t(1-t)=s(1-s)$, whence $s=t$ or $1-t$. This completes the proof of (2.4).

We note this consequence. See (3.2) also.

(2.5) If the scalar field $k$ is infinite and $p^{e_{1}} \neq 2$, (see (2.1)), then there are infinitely many odd-dimensional indecomposable G-modules of height three.

The remarks (1.5) on faithfulness in the situation $G$ is elementary abelian, $p$ odd, apply here as well.

(2.6) The dual module to $V_{t}$. Now we define a left action of $k G$ on $\operatorname{Hom}_{k}\left(V_{t}, k\right)$ by $(a * f)(x)=f(a x)$ for $a \in k G, f \in \operatorname{Hom}_{k}\left(V_{t}, k\right)$, and $x \in V_{t}$. Note that $(a b) * f=a *(b * f)$ because $k G$ is commutative. We denote the module thus obtained $V_{t}^{*}$.

(Note also that a different left action may be obtained by using the natural involution $a \mapsto a^{-1}$ of $G$, rather than commutativity.)

Now let $\left\{f_{i}\right\}$ be the $k$-basis for $V_{t}^{*}$ dual to $\left\{x_{i}\right\}$, so that $f_{i}\left(x_{j}\right)=\delta_{i j}$. Then the assignment

$$
f_{1} \mapsto x_{2 n+1}, \cdots, f_{n+1} \mapsto x_{n+1}, \cdots, f_{2 n+1} \mapsto x_{1}
$$

extends to a $k G$-isomorphism $V_{t}^{*} \widetilde{\rightarrow} V_{1-t}$.

We conclude, using (2.4), that $V_{t}$ is isomorphic with its dual iff $t \neq 0,1$.

\section{Abelian $p$-groups of higher exponent.}

In this section we construct families of indecomposables for abelian $p$-groups of exponent greater than $p$.

Let $G, k$ be as in (1.1). Let $U$ denote the $p$-group of unipotent matrices over $k$ of the form $I+N$, with $N$ strictly lower triangular. Any representation $G \rightarrow$ Aut $_{k} V$ may, by proper choice of basis, be viewed as a homomorphism $\rho: G \rightarrow U$. The group $U$ contains an element of order $p^{d}$ iff $\operatorname{dim}_{k} V \geq p^{d-1}+1$. Furthermore $V$ can be made indecomposable with respect to such an element iff $\operatorname{dim}_{k} V \leq p^{d}$. For the time being, assume exponent $G=p^{d}$ and $p^{d-1}+1 \leq \operatorname{dim}_{k} V \leq p^{d}$, and that, by a further modification of basis if necessary, the representation $\rho: G$ $\rightarrow U$ satisfies 


$$
\rho\left(a_{1}\right)=\left(\begin{array}{llll}
1 & & & 0 \\
1 & & & \\
& \ddots & & \\
0 & & 1 & 1
\end{array}\right) \quad \text { (in Jordan form) }
$$

The centralizer $\Gamma$ in $U$ of $\rho\left(a_{1}\right)$ is the subgroup of $U$ of matrices $\left(\alpha_{i j}\right)$ with constant subdiagonals : $\alpha_{i j}=\alpha_{i+1, j+1}$ for $i>j . \quad(\Gamma=I+$ (commutative algebra generated over $k$ by $\left.\rho\left(a_{1}\right)-I\right)$.) Thus $\rho(G) \subset \Gamma$. By letting the first non-zero subdiagonal be the $r$-th for appropriate $r$, one gets a matrix of any order $p^{\nu}, \nu \leq d$. Further, if $a_{2}$ is a second generator of $G$ of order $p^{\nu}$, choosing distinct constant entries $t \in k$ for the $r$-th subdiagonal yields inequivalent modules $V_{t}$ (here $\rho\left(\alpha\left(a_{1}-1\right)^{r}-\left(a_{2}-1\right)\right.$ ) annihilates $V_{t} /\left(a_{1}-1\right)^{r+1} V_{t}$ iff $\left.\alpha=t\right)$. Since the appropriate location of the constant subdiagonals is sufficient to determine a representation, we see also that $\rho$ can be made faithful if $k$ is sufficiently large and exponent $G=p^{d}$.

We combine the representations of Sections 1 and 2 with the above described minimal faithful ones $V_{t}$.

(3.1) Proposition. Let $\left\{v_{1}, \cdots, v_{m}\right\}, m \geq 3$, be a basis of $V_{t}$ giving the desired lower triangular form. Let $M$ be an indecomposable G-module with cyclic submodule $M_{0}$ isomorphic via $\varphi$ to some submodule $k$-span $\left\{v_{\lambda}, \cdots, v_{m}\right\}, \lambda \geq 2$, of $V_{t}$. Assume $\left(a_{1}-1\right)^{m-\lambda+1}(M)=(0)$. Then

$$
W=V_{t} \oplus M / k-\operatorname{span}\left\{v_{\lambda}-\varphi^{-1}\left(v_{\lambda}\right), \cdots, v_{m}-\varphi^{-1}\left(v_{m}\right)\right\}
$$

is indecomposable.

Proof. We denote the images in $W$ of the $v_{i}$ by $\bar{v}_{i}$. If $\bar{v}_{1}+w \in Z$, a submodule of $W$, then $\left(a_{1}-1\right)^{m-1}\left(\bar{v}_{1}+w\right)=\bar{v}_{n} \in Z$. Let $W=Z_{1} \oplus Z_{2}$ and suppose some $\bar{v}_{1}+w \in Z_{1}$. Then as vector space we have a nontrivial decomposition

$$
Z_{1}=k-\operatorname{span}\left\{\left(a_{1}-1\right)^{j}\left(\bar{v}_{1}+w\right): j=0, \cdots, \lambda-2\right\} \oplus\left(Z_{1} \cap \bar{M}\right),
$$

where $\bar{M}$ denotes the image of $M$ in $W$. Since $\bar{v}_{j}+w^{\prime} \in Z_{2}, j<\lambda$, implies $\bar{v}_{m} \in Z_{2}$, we have $Z_{2} \subset \bar{M}$. Thus $\bar{M}$ is decomposed. Since $\bar{M}$ is isomorphic to $M, Z_{2}=(0)$.

In this situation, given non-isomorphic $V_{t}$ or non-isomorphic $M$ 's, the resulting modules $W$ are non-isomorphic. In fact, then $\bar{V}_{t}$ is the only $m$-dimensional cyclic submodule of $W$, while $\bar{M}=\operatorname{ker}\left(a_{1}-1\right)^{m-\lambda+1}$. 
We may apply (3.1) using for $M$ the second odd-dimensional class $\left(M_{0}=k\right.$-span $\left\{x_{n+1}, x_{2 n+1}\right\}$ with $\left(a_{i}-1\right)\left(x_{n+1}\right) \in k$-span $\left.\left\{x_{2 n+1}\right\}, i>2\right)$ provided $\left(a_{2}-1\right) v_{m-1}=0$ in $V_{t}$. This can be arranged if either $a_{2}$ has order $<p^{d}$ or $n>p^{d-1}+1$. We may also take $M$ in the even-dimensional class, with $M_{0}=k$-span $\left\{x_{n}, x_{2 n}\right\}$ and $\left(a_{i}-1\right)\left(x_{n}\right) \in k$-span $\left\{x_{2 n}\right\}, i>2$. Note that the choice of $t \in k$ for the action of $a_{2}-1$ is already determined by $V_{t}$.

One may also take for $M$ one of the modules of Section 2, with $M_{0}=k$-span $\left\{x_{n+1}, x_{2 n+1}\right\}$ and appropriate choices for action of the $a_{i}-1$, $i>2$, provided again that $\left(a_{2}-1\right) v_{m-1}=0$, and $m>3$. In this case, however, the hypothesis that $\left(a_{1}-1\right)^{m-\lambda+1}(M)=0$ fails, and a slightly more complicated argument specific to $M$ is needed to prove $W$ indecomposable. For other $M$, and in the absence of that hypothesis, $W$ may decompose.

A partial summary of the material of Sections 1,2 , and 3 is the following :

(3.2) THEOREM. With the exception of $C(2) \times C(2)$, a non-cyclic abelian p-group has infinitely many indecomposables in every dimension $\geq 2$ over an infinite field of characteristic $p$. If $p^{d}$ is the exponent of the group, these representations may be taken faithful in dimensions $\geq p^{d-1}+1$.

\section{The anomalous case: The four-group in characteristic 2.}

In this section we show that the procedure of Section 2 for obtaining an infinite family of odd-dimensional indecomposables fails for $G=$ $C(2) \times C(2)$. In fact we show essentially that in this case neither of the two odd-dimensional Heller-Reiner modules is a member of any non-trivial larger parametrized family of modules, indecomposable or decomposable, unless the group algebra $k G$ is a direct summand of the generic members of that family. Our argument involves straightening out a deformation, and the method is reminiscent of Dieudonné's in [4]. Consequently a portion of his characterization of the odd-dimensional indecomposables in this situation emerges from our proof.

A complete statement of results is as follows:

(4.1) THEOREM. Let $G$ be the four-group, and $V$ be an indecomposable G-module of odd dimension $\geq 3$ over a field $k$ of characteristic 2 . Then

(i) every non-trivial generic deformation $V_{t}$ of $V$ decomposes directly 
over $K G$; in fact, $V_{t} \simeq K G \oplus W$, for some $K G$-submodule $W$; and

(ii) $V$ is a Heller-Reiner module of the first (1.2) or second (1.3) class.

Here $K$ is the power series field $k((t))$. For deformations, we employ only some definitions and two lemmas from [6]. Statement (ii) is of course known. We use some weaker consequences of it, Lemmas (4.2) and (4.3) below, in the proof of (i). This proof shows (ii) to be a consequence of (4.2) and (4.3).

We have $G=\{1, x, y, x y\}$, and write $\alpha=1-x, \beta=1-y$ in the group algebra $k G$. Thus $\operatorname{rad} k G$ has basis $\alpha, \beta, \alpha \beta$. Suppose $\varphi: k G \rightarrow \operatorname{End}_{k} V$ gives the odd-dimensional indecomposable representation. Then it is wellknown that $\varphi(\alpha \beta)=0$, for otherwise $V$ would contain a submodule isomorphic to the free (and hence injective) left regular module $k G$, which would therefore split off from $V$.

Likewise, if $\varphi_{t}=\varphi+t \Phi_{1}+t^{2} \Phi_{2}+\cdots$ affords a deformation $V_{t}$ such that $\varphi_{t}(\alpha \beta) \neq 0$, then $V_{t}$ has a $K G$-direct summand isomorphic with $K G$. (Here the underlying space for $V_{t}$ is $V_{K}$, obtained by extending scalars to the power series field $K$. This procedure is standard; see [6]).

It remains to show, therefore, that every deformation $\varphi_{t}$ with the property $\varphi_{t}(\alpha \beta)=0$ is a trivial deformation. This is true in any characteristic. See (4.4) below.

Since $\alpha \beta$ will act as zero from now on, we may take $\varphi$ and $\varphi_{t}$ as representations of the 3-dimensional quotient algebra $k[a, b]=k G /(\alpha \beta)$, where $a=\alpha+(\alpha \beta)$ and $b=\beta+(\alpha \beta)$. Note $N=\operatorname{rad} k[a, b]$ is square zero on $a$ and $b$.

Denoting $\varphi(N) V$ by $Y$, we may write $V=X \oplus Y$, where $X$ is a $k$ space complement to $Y$. Since $\varphi(a) Y=\varphi(b) Y=(0)$, we may as well consider the operators $\varphi(a)$ and $\varphi(b)$ on $V$ as mappings $X \rightarrow Y$.

(4.2) Lemma. If $V$ is indecomposable as a $k[a, b]-m o d u l e$ and if $\operatorname{dim}_{k} X \leq \operatorname{dim}_{k} Y$, then both mappings $\varphi(a), \varphi(b): X \rightarrow Y$ are injective.

$V$ must in fact have one of the structures given in (1.2) and (1.4). From (4.1) (ii) we also have

(4.3) LEMMA. Let $V$ be a $(2 n+1)$-dimensional indecomposable module for $k[a, b]$. Then $\operatorname{dim}_{k} \varphi(N) V$ equals $n$ or $n+1$.

Now all of (4.1) follows from (4.4) using only (4.2) and (4.3). 
(4.4) LEMMA. Let $k$ be any field, and $V$ a $(2 n+1)$-dimensional indecomposable module for the algebra $k[a, b]$ afforded by the representation $\varphi$. Then

(i) $V$ is rigid as a $k[a, b]-m o d u l e$;

(ii) if $\operatorname{dim}_{k} \varphi(N) V=n+1$, then $V$ has a k-basis $x_{1}, \cdots, x_{n}, y_{1}, \cdots$, $y_{n+1}$ such that, for $1 \leq i \leq n$,

$$
\varphi(a) x_{i}=y_{i}, \quad \varphi(b) x_{i}=y_{i+1} ;
$$

(iii) otherwise, $V$ is the $n$-dimensional $k$-dual of the module in (ii).

It is straightforward that (iii) follows from (ii), (4.3) and Lemma 4 of [6]. To prove (i) and (ii), suppose we are given a deformation $V_{t}$ of $V$ afforded by a representation $\varphi_{t}=\varphi+t \Phi_{1}+\cdots$ of $K[a, b]$. We assert first that if $Y=\varphi(N) V$ has dimension $n+1$ over $k$, then $Z=$ $\varphi_{t}\left(N_{K}\right) V_{K}$ has dimension $n+1$ over the power series field $K$ and, moreover, $Z=\operatorname{ker} \varphi_{t}(a)=\operatorname{ker} \varphi_{t}(b)$. Here we are considering $\varphi_{t}(a)$ and $\varphi_{t}(b)$ as operators on $V_{t}$. To see this, note first that $Z$ is contained in both kernels because $a b=b a=0$. Moreover, each kernel has $K$-dimension $n+1$, because any square zero operator on $V_{K}$ has a kernel of dimension $\geq n+1$, while Lemma 8 of [6] says that $\operatorname{dim}_{K} \operatorname{ker} \varphi_{t}(a)$ is less than or equal to $\operatorname{dim}_{k} \operatorname{ker} \varphi(a)$. Thus $\operatorname{dim}_{K} Z \leq n+1$. On the other hand, $Z$ has $K$-dimension at least as large as $\operatorname{dim}_{k} \varphi(N) X=n+1$, again by Lemma 8 of [6].

It follows that the $k$-space decomposition $V=X \oplus Y$ has a counterpart $V_{t}=X_{K} \oplus Z$ over $K$. We next assert that $X_{K}$ has a $K$-space basis $\xi_{1}, \cdots, \xi_{n}$ where $\xi_{i}=\xi_{i}(t)=x_{i_{0}}+t x_{i_{1}}+\cdots$ (cf. order zero) with the properties that $x_{10}, \cdots, x_{n 0}$ is a $k$-basis for $X$ and also $\varphi_{t}(b) \xi_{i}=\varphi_{t}(a) \xi_{i+1}$ for $i=1, \cdots, n-1$ when $n \geq 2$. We go by induction on $n$, noting that the case $n=1$ is clear.

To begin the induction process, define

$$
Z_{1}=\left(\varphi_{t}(a) X_{K}\right) \cap\left(\varphi_{t}(b) X_{K}\right), \quad \Xi=\left.\varphi_{t}(a)\right|_{X_{K}} ^{-1} Z_{1} .
$$

Note that $Z_{1}$ is of codimension 2 in $Z$, whence $E$ is of codimension 1 in $X_{K}$. Now let $W$ be the submodule of $V_{t}$ generated over $K[a, b]$ by the subspace $\boldsymbol{E}$,

$$
W=\Xi+\varphi_{t}(a) \Xi+\varphi_{t}(b) \Xi
$$

and, having this, let $W_{0}$ be the $k[a, b]$-submodule of $V$ generated by the 
"constant terms" in $W$; see Lemma 5 in [6]. It follows that

$$
W_{0}=\Xi_{0}+\varphi(a) \Xi_{0}+\varphi(b) \Xi_{0}
$$

where $\Xi_{0}$ is the $k$-space of constant terms in $\Xi$. Note $\operatorname{dim}_{k} W_{0} \geq 2(n-1)$, since $\operatorname{dim}_{k} \Xi_{0}=n-1$.

We assert that in fact $\operatorname{dim}_{k} W_{0}=2 n-1$ and that $W_{0}$ is an indecomposable $k[a, b]$-module. First the assumption $\operatorname{dim}_{k} W_{0}=2(n-1)$ would imply $\varphi(a) \boldsymbol{E}_{0}=\varphi(b) \boldsymbol{\Xi}_{0}$. Then any $x \in X, x \notin \boldsymbol{E}_{0}$, would generate a 3-dimensional $k[a, b]$-module complement to $W_{0}$ in $V$, a contradiction. Now given a module decomposition $W_{0}=T \oplus T^{\prime}$, assume $T^{\prime}$ even-dimensional. From previous remarks $\left.\left.\operatorname{ker} \varphi(a)\right|_{W_{0}} \cap \operatorname{ker} \varphi(b)\right|_{W_{0}}$ is $\varphi(N) W_{0}$. Thus we have $T^{\prime}=X^{\prime} \oplus Y^{\prime}$ where $X^{\prime} \subset X$ and $Y^{\prime}=\varphi(N) T^{\prime} . \quad$ A similar decomposition of $T$ implies $\operatorname{dim} \varphi(N) T>\frac{1}{2} \operatorname{dim} T$, whence $\operatorname{dim} X^{\prime}=\operatorname{dim} Y^{\prime}$, and $\left.\varphi(a)\right|_{X^{\prime}}$ and $\left.\varphi(b)\right|_{X^{\prime}}$ are isomorphisms. Let $X^{\prime \prime} \oplus X^{\prime}=X$. Then $\left[\varphi(N) X^{\prime \prime}\right]$ $\cap Y^{\prime}=(0)$ by dimensionality. Thus $T^{\prime}$ has a complement $X^{\prime \prime}+\varphi(N) X^{\prime \prime}$ in $V$ and so is (0).

Now by induction, there exists a $K$-basis $\xi_{2}, \cdots, \xi_{n}$ for $\Xi$ such that each $\xi_{i}$ has the form $x_{i_{0}}+t x_{i_{1}}+\cdots$ and also $\varphi_{t}(b) \xi_{i}=\varphi_{t}(a) \xi_{i+1}, i=2$, $\cdots, n-1$. Recalling the definition of $E$, we note that $\varphi_{t}(a) \xi_{2}, \cdots, \varphi_{t}(a) \xi_{n}$ is a basis for $Z_{1}$. Since $Z_{1} \subset \varphi_{t}(b) X_{k}$, we may complete our basis of $X_{k}$ by choosing a series $\xi_{1}$ of order 0 in $X_{K}$ such that $\varphi_{t}(b) \xi_{1}=\varphi_{t}(a) \xi_{2}$. This gives us the basis of $\xi_{i}$ 's as asserted.

Now we complete the proof of (i) and (ii) in the statement of (4.4) by defining $x_{i}=x_{i_{0}}$ and $y_{i}=\varphi(a) x_{i}$ for $1 \leq i \leq n$, and also $y_{n+1}=\varphi(b) x_{n}$. To show that the deformation $V_{t}$ is trivial we define the mapping $I_{t}: V_{K}$ $\rightarrow V_{K}$ (a deformation of the identity mapping of the underlying $K$-space; see [6, Lemma 3]) by

$$
I_{t}\left(x_{i}\right)=\xi_{i}, \quad I_{t}\left(y_{i}\right)=\varphi_{t}(a) \xi_{i}, \quad I_{t}\left(y_{n+1}\right)=\varphi_{t}(b) \xi_{n}
$$

for $1 \leq i \leq n$. One checks readily that $I_{t}$ affords an equivalence of the representations $\varphi$ and $\varphi_{t}$, whence the deformation $\varphi_{t}$ of $\varphi$ is trivial. This completes the proof of Lemma (4.4) and thus of Theorem (4.1).

\section{Some examples of deformations in the even-dimensional case.}

In Section 4 we did not determine whether for $G=C(2) \times C(2)$, the $K G$-modules of types (1.2) and (1.3) can deform so as to acquire a summand isomorphic to $K G$. In fact we intend to give a fuller treatment 
of this problem later in which we show this to be impossible. There the Hochschild cohomology is a useful computational device.

For the present we content ourselves with two examples to illustrate the deformation structure of the even-dimensional Heller-Reiner modules for $G=C(2) \times C(2)$ in characteristic two.

(5.1) The 4-dimensional module of type (1.4) with $t=0$ deforms into the left regular module $K G$.

We let $u$ represent the variable in the power series field $K$. Then defining

$$
\varphi_{u}\left(a_{2}-1\right)\left(x_{1}\right)=x_{4}+u x_{2}, \quad \varphi_{u}\left(a_{2}-1\right)\left(x_{3}\right)=u x_{4},
$$

with other action as before, gives the result. For now $x_{1}$ acts as cyclic generator.

Actually here again one may show that for $t \neq 0$, there is no deformation into $K G$, nor is there even a deformation with a summand isomorphic to $K G$ in the higher dimensional cases of (1.4) with $t \neq 0$. One might say that the kernel of these $K G$-representations is rigid.

(5.2) All the modules (1.4) for $G=C(2) \times C(2)$ admit decomposable generic deformations of height two.

We factor through the algebra $k[a, b]$ of Section 4 , letting $a$ act as $a_{1}-1$ and $b$ as $a_{2}-1$. Hence the following is valid in any characteristic. With the given action of $a$, and an arbitrary map $\varphi(b): X \rightarrow Y$, the module $X \oplus Y$ is indecomposable if and only if with respect to the given bases of $X$ and $Y$ the map $\varphi(b): X \rightarrow Y$ has an indecomposable matrix. It suffices then to give a deformation preserving the action of $a$ and such that $\varphi_{u}(b): X \rightarrow Y$ has a matrix with distinct eigenvalues in $K$. For example, we may define

$$
\varphi_{u}(b)\left(x_{r}\right)=\varphi(b)\left(x_{r}\right)+u^{r} x_{r+n}, \quad 1 \leq r \leq n .
$$

Note that if $\varphi_{u}(b)$ has distinct eigenvalues only in the algebraic closure of $K$ then the generic deformation might actually be indecomposable over $K$ in spite of the fact that all specializations of $u$ into $k$ might yield decomposable modules. One may often avoid this difficulty by replacing $u$ by a suitable power $u^{N}$ before making the deformation. 


\title{
REFERENCES
}

[1] V. A Basev, Representations of the group $Z_{2} \times Z_{2}$ into a field of characteristic 2, Dokl. Akad. Nauk. SSSR 141 (1961), 1015-1018.

[ 2 ] S. B. Conlon, Certain representation algebras, J. Aust. Math. Soc. 5 (1965), 83-99.

[ 3 ] C. Curtis and I. Reiner, Representation theory of finite groups and associative algebras, Interscience, New York, 1962.

[ 4 ] J. Dieudonné, Sur la réduction canonique des couples de matrices, Bull. Soc. Math. France 74 (1946), 130-146.

[5] J. D. Donald and F. J. Flanigan, A deformation-theoretic version of Maschke's theorem for modular group algebras: the commutative case, J. Algebra 29 (1974), 98-102.

[ 6 ] — Deformations of algebra modules, J. Algebra 31 (1974), 245-256.

[ 7 ] - Parameter varieties of finite group representations, In preparation.

[ 8 ] A. Heller and I. Reiner, Indecomposable representations, Ill. J. Math. 5 (1961), $314-323$.

[ 9 ] D. Higman, Indecomposable representations at characteristic $p$, Duke Math. J. 21 (1954), 377-381.

[10] G. J. Janusz, Faithful representations of $p$-groups at characteristic $p$, Representation Theory of Finite Groups and Related Algebras, Proceedings of Symposia in Pure Mathematics 21 (1970), 89-90.

[11] —, Faithful representations of $p$-groups at characteristic $p$, II, J. Algebra 22 (1972), 137-160.

[12] D. L. Johnson, Indecomposable representations of the four-group over fields of characteristic 2, J. London Math. Soc. 44 (1969), 235-298.

\author{
Birchwood \\ Tyringham, Mass. \\ Department of Mathematics \\ San Diego State University
}

